\title{
COSTURANDO SABERES NA COLCHA DE RETALHOS: ALINHAMENTO CONCEITUAL NO PET-SAÚDE INTERPROFISSIONALIDADE EM UMA UNIDADE SAÚDE DA FAMÍLIA
}

\author{
SEWING KNOWLEDGE IN THE PATCHWORK: CONCEPTUAL \\ ALIGNMENT IN PET-HEALTH INTERPROFESSIONALITY IN A FAMILY \\ HEALTH UNIT.
}

Cristiane Costa Braga ${ }^{1}$

RESUMO: Objetivo: Relatar a atividade de alinhamento conceitual e metodológico, sobre Educação Interprofissional (EIP) e Práticas Colaborativas (PC) com as atividades do PET-Saúde Interprofissionalidade da UFPB/SMS em uma Unidade Saúde da Família (USF). Método: Utilizou-se a dinâmica da "colcha de retalhos", construída com registros fotográficos das atividades realizadas e targetas com os conceitos da EIP e PC, fundamentada por metodologia ativa, com destaque na problematização das atividades desenvolvidas em uma USF, em uma aprendizagem significativa baseada na prática e diálogo entre os estudantes da graduação, os preceptores (profissionais dos serviços) e os tutores (docentes da Instituição de Ensino Superior - IES), inseridos em grupos de aprendizagem tutorial no PET-Saúde Interprofissionalidade. Resultados: Permitiu a imersão dos estudantes no cenário da Atenção Primária a Saúde, possibilitando vivenciar as atividades clínicas e coletivas, promovendo um aprendizado acerca da cartografia do território, do diagnóstico situacional, planejamento das ações em equipe interprofissional, realização de Educação em Saúde, Educação Permanente, discussão de casos clínicos e apreensão dos conceitos acerca da EIP e PC. Conclusão: A dinâmica abordada colaborou para a reflexão das atividades de planejamento, construção, execução e avaliação em equipe interprofissional, à luz dos conceitos da EIP e PC, favorecendo o compartilhamento de saberes entre profissionais da Saúde, da Educação e os estudantes da Graduação, tornando possível a aquisição de conhecimentos nas temáticas abordadas para a qualificação do cuidado em saúde da comunidade, em uma ação intencional de aprender uns com os outros, sobre os outros e entre si, fortalecendo a articulação ensino- serviço-comunidade.

\footnotetext{
${ }^{1}$ Cirurgiã Dentista na Estratégia Saúde da Família. Mestra em Saúde da Família. Preceptora do PETSaúde Interprofissionalidade UFPB/SMS - João Pessoa.
} 
Palavras chave: Estratégia Saúde da Família. Educação Interprofissional. Serviços de Integração Docente-Assistencial.

ABSTRACT: Objective: To report the conceptual and methodological alignment activity on Interprofessional Education (IPE) and Collaborative Practices (PC) with the activities of PET-Health Interprofessionality of UFPB / SMS in a Family Health Unit (USF). Method: The dynamics of the "patchwork" was chosen, built with photographic records of the activities performed and targeted with the concepts of IPE and PC, based on active methodology, highlighting the problematization of activities developed in a USF, in a learning process based on the significant dialogue between undergraduate students, preceptors (service professionals) and tutors (teachers of the Higher Education Institution - HEI), inserted in tutorial learning groups at PET-Health Interprofessionality. Results: It allowed the immersion of students in the Primary Health Care scenario, enabling the experience of clinical and collective activities, promoting a learning about the cartography of the territory, the situational diagnosis, planning of actions in the interprofessional team, conducting Health Education, Education Permanent, discussion of clinical cases and apprehension of the concepts about IPE and CP. Conclusion: The dynamics approached contributed to the reflection of the planning, construction, execution and evaluation activities in an interprofessional team, in light of the concepts of IPE and $C P$, favoring the sharing of knowledge between Health professionals, Education and undergraduate students and making possible the acquisition of knowledge in the themes addressed for the qualification of community health care, in an intentional action to learn from each other, about each other and among themselves, strengthening the teaching-service-community articulation.

Keywords: Family Health Strategy, Interprofessional Education, Teaching Care Integration Services. 


\section{INTRODUÇÃO}

O Sistema Único de Saúde (SUS) está pautado nos princípios e diretrizes da universalidade, equidade, integralidade, descentralização, participação popular e organizado pela rede de serviços de modo regionalizado e hierarquizados. Configura a política pública mais inclusiva realizada no Brasil, e seu avanço mais significativo ocorreu na Atenção Primária a Saúde (APS) (BRASIL, 2016; MENDES, 2019).

Neste contexto, a APS se configura como o conjunto de ações de saúde individuais e coletivas, envolvendo atividades de promoção, prevenção, proteção, diagnóstico, tratamento, reabilitação, redução de danos, cuidados paliativos e vigilância em saúde, desenvolvida através de práticas de cuidado integrado e gestão qualificada, realizada com equipe multiprofissional. Com o objetivo de reorganizar esse ponto de atenção à saúde, criou-se a Estratégia Saúde da Família (ESF) com atividades que requerem atuação integrada e colaborativa de um amplo elenco de profissionais de saúde, como o profissional médico, agentes comunitários de saúde, enfermeiros, auxiliares e técnicos de enfermagem, cirurgiões dentistas, auxiliares e técnicos de saúde bucal, bem como os profissionais inseridos nos Núcleos de Apoio à Saúde da Família (NASF), a exemplo dos fisioterapeutas, fonoaudiólogos, terapeutas ocupacionais, educadores físicos, psicólogos entre outros, tendo portanto, o caráter eminentemente interprofissional da atenção à saúde e da formação dos profissionais (BRASIL, 2017a; PEDUZZI, 2016).

A definição de EIP mais amplamente conhecida é a do Centro para o Avanço da Educação Interprofissional em Saúde (CAIPE), situado no Reino Unido, que a define enquanto situação em que membros de duas ou mais profissões aprendem com, sobre e entre si, para melhorar a colaboração e a qualidade da atenção à saúde (CAIPE, 2002).

Outrossim, a Organização Mundial de Saúde - OMS (2010) incorporou tal conceito, acrescentando que a EIP ocorre quando estudantes de duas ou mais 
profissões aprendem sobre os outros, com os outros e entre si, para possibilitar a colaboração eficaz e melhorar os resultados na saúde.

Educação Interprofissional, sendo assim, se concretiza quando os profissionais de saúde aprendem colaborativamente dentro de suas áreas e através de outras profissões, com a finalidade de obter conhecimentos, habilidades e valores necessários para trabalhar com outros profissionais de saúde (CIHC, 2010). Esta proposta leva em consideração que os conhecimentos, as habilidades e os valores para trabalhar com outros profissionais de saúde devem ser desenvolvidos no processo formativo de uma profissão e entre diferentes áreas profissionais, tendo a colaboração como um elemento também presente nessa aprendizagem (COSTA et al 2018).

Reeves et al (2016a) afirma que membros de duas ou mais profissões aprendem em conjunto, de forma interativa, com o propósito explicito de melhorar a qualidade da atenção e o bem-estar do usuário, família e comunidade. Sob o mesmo prisma, Barr e Low (2013) destacam que a aprendizagem interprofissional está baseada no trabalho, podendo acontecer sempre que duas ou mais profissões trabalham juntas.

Nesse sentido, a Educação Interprofissional (EIP), em suas bases teóricas e metodológicas, complementa e solidifica os ideários do SUS, fornecendo subsídios para a construção de um projeto novo de sociedade, a partir da concepção ampliada de saúde (COSTA, 2016).

Resultados de pesquisa direcionam para a importância do trabalho interprofissional e da integração ensino-serviço-comunidade, o que colabora para aproximar o estudante da realidade social e sanitária da população e do processo de trabalho dos serviços de atenção primária à saúde, ultrapassando os limites da teoria por meio do contato com o cotidiano das Unidades de Saúde das Famílias (USF) (MADRUGA et al 2015).

Costa (2016) relata que o fortalecimento e consolidação da EIP necessita de clareza no reconhecimento e na intencionalidade da prática colaborativa. Faz-se necessário também entender que a prática colaborativa na atenção à saúde deveria ocorrer sempre que profissionais de saúde de diferentes áreas prestassem serviços com base na integralidade da saúde, envolvendo os pacientes e suas famílias, 
cuidadores e comunidades para atenção à saúde da mais alta qualidade em todos os níveis da rede de serviços, incluindo o trabalho clínico e não clínico relacionado à saúde, como diagnóstico, tratamento, vigilância, comunicação em saúde, administração e engenharia sanitária (OMS, 2010).

A colaboração implica em uma relação/interação permanente entre os trabalhadores de saúde, mas também atribuindo a centralidade do usuário, família e comunidade para a produção de serviços de saúde de melhor qualidade. Dessa forma, tais parcerias podem inclusive constituir formas de resistência às ameaças de retrocesso nas políticas de saúde que constituíram e consolidaram o SUS e a ampliação de acesso aos serviços de APS (COSTA et al 2018; PEDUZZI, 2018).

Um dos benefícios da implementação da Educação Interprofissional e da Prática Colaborativa é que essas estratégias trazem mudanças na forma como os profissionais de saúde se relacionam uns com os outros para prestar assistência, e, dessa maneira, ao modificar a forma como os profissionais de saúde pensam e interagem, a cultura do ambiente laboral e as atitudes da força de trabalho se transformarão, melhorando a vivência trabalhista dos profissionais, trazendo benefícios para a comunidade como um todo (OMS, 2010).

Para tanto, seria preciso o desenvolvimento de competências comuns à prática colaborativa, tais como: a comunicação interprofissional, o cuidado centrado no paciente, a clareza de papéis dos profissionais, o funcionamento da equipe, a resolução de conflitos e liderança colaborativa (CIHC, 2010).

No Brasil, esforços são feitos para construir uma definição de educação interprofissional coerente com os desafios enfrentados pela realidade da formação dos profissionais de saúde (BRASIL, 2018a). Batista (2012) descreve que vivências e relatos de experiências de treinamento conjunto e aprendizagens compartilhadas em políticas indutoras de mudanças na graduação como o Pró-Saúde e o PETSaúde têm-se revelado como potenciais espaços de EIP.

O Programa de Educação pelo Trabalho para a Saúde (PET-Saúde), uma parceria dos Ministérios da Saúde (MS) e o Ministério da Educação (ME), é destinado a fomentar grupos de aprendizagem tutorial em áreas estratégicas para SUS, tendo como pressuposto a educação pelo trabalho, caracterizando-se como instrumento para qualificação em serviço dos profissionais da saúde, bem como de 
iniciação ao trabalho, dirigido aos estudantes dos cursos de graduação e de pósgraduação na área da saúde (BRASIL, 2010).

No ano de 2018, foi lançado o edital de seleção do PET-Saúde Interprofissionalidade, convocando as Instituições de Ensino Superior (IES) em parcerias com as Instituições de Serviços de Saúde a construírem projetos que fomentasse mudanças curriculares alinhadas às Diretrizes Curriculares Nacionais (DCNs) para todos os cursos de graduação na área da saúde, levando-se em consideração estratégias correlacionadas aos princípios da interprofissionalidade, interdisciplinaridade e intersetorialidade, como fundamentos da mudança, na lógica da formação dos profissionais e na dinâmica da produção do cuidado em saúde, visando a proporcionar qualificação dos processos de integração ensino-serviçocomunidade, de forma articulada entre o SUS e as IES, de modo a promover a Educação Interprofissional e as Práticas Colaborativas em saúde (BRASIL, 2018b).

A UFPB, em parceria com Secretaria Municipal da Saúde (SMS) de João Pessoa, teve seu projeto aprovado para o Programa de Educação pelo Trabalho para a Saúde PET-Saúde- Interprofissionalidade - 2018/2019, nos termos do Edital SGTES/MS no 10 , de 23 de julho de 2018, o qual contemplaria suas atividades nas Unidades Saúde da Família (USF) deste Município (BRASIL, 2018c).

Este trabalho objetiva relatar a atividade de alinhamento conceitual $\mathrm{e}$ metodológico, sobre Educação Interprofissional e Práticas Colaborativas com as atividades do PET-Saúde Interprofissionalidade da UFPB/SMS em uma USF. Foi realizada pelos preceptores, estudantes da graduação e tutores participantes deste Projeto, utilizando a dinâmica da "colcha de retalhos", fundamentada na metodologia ativa, com destaque na problematização das atividades desenvolvidas nos serviços de saúde, em uma aprendizagem significativa baseada na prática e diálogo entre os participantes, bem como, evidenciando a contribuição destas vivências no processo do cuidado e na formação em saúde. 


\section{RELATO DE EXPERIÊNCIA}

\section{Conhecendo o PET-Saúde Interprofissionalidade UFPB/SMS}

A inserção dos estudantes na APS é importante para que eles conheçam a dimensão complexa do trabalho em saúde na comunidade, as necessidades de saúde, além de aperfeiçoar a capacidade de crítica e reflexão (BRASIL, 2018a).

As atividades do PET-Saúde Interprofissionalidade UFPB/SMS-JP são desenvolvidas por tutores (docentes da Instituição de Ensino Superior - IES), preceptores (profissionais de saúde das Unidades Saúde da Família) e os estudantes dos cursos de graduação inseridos em grupos de aprendizagem tutorial, cumprindo uma carga horária estabelecida em edital de oito horas semanais. Neste contexto, foi constituído o Grupo de Trabalho (GT) na APS, em que a equipe de preceptores e estudantes está inserida em cenários de aprendizagem nas Unidades de Saúde especificamente na Estratégia Saúde da Família, objetivando fortalecer mudanças na formação em saúde de acordo com as necessidades de saúde para o SUS e qualificação da integração ensino-serviço-comunidade. O GT também realiza reuniões no Campus I da UFPB, objetivando formação pedagógica de modo a promover a Educação Interprofissional e as Práticas Colaborativas em saúde, bem como permitir o compartilhar de saberes e a aprendizagem com os demais participantes do Projeto. Estes grupos de aprendizagem tutorial são acompanhados pela Coordenação de Gestão Local do Projeto, a qual está vinculada à Coordenação Geral da Secretaria de Gestão do Trabalho e da Educação na Saúde, do Ministério da Saúde (SGTES/MS).

Nesse relato de experiência, as atividades do GT foram realizadas na Unidade Saúde da Família Integrada Verdes Mares, cuja equipe de trabalho está composta por dois estudantes da graduação, sendo uma do curso de Terapia Ocupacional e outro do curso de Medicina, uma preceptora, que é a profissional Cirurgiã Dentista da Estratégia Saúde da Família, sob a supervisão de duas Tutoras, 
docentes da UFPB dos cursos de Odontologia e Fonoaudiologia, seguindo a recomendação do edital de seleção do PET-Saúde Interprofissionalidade e obedecendo aos critérios estabelecidos na seleção pública destes participantes. As atividades em campo tiveram início no mês de junho de 2019 e encontram-se em andamento, no segundo semestre de atividades do Projeto.

As reuniões iniciais foram no sentido de apresentação e apropriação dos objetivos e metodologias do Projeto, aproximação dos participantes dos Grupos de Trabalho e dos Grupos Tutorial, bem como leitura e discussão de artigos sobre Educação Interprofissional, Práticas Colaborativas, Trabalho Interprofissional, entre outros.

Simultaneamente às ações que aconteciam nos grupos de trabalho e nos grupos tutoriais, ocorriam encontros presenciais com todos os participantes, como também ocorriam reuniões da Comissão Colegiada Local e com os representantes do grupo assessor do Projeto, fortalecendo e direcionando as ações planejadas, conforme previsto no edital do PET-Saúde Interprofissionalidade (BRASIL, 2018b).

A imersão dos estudantes, participantes do PET-Saúde Interprofissionalidade no cenário de aprendizagem na Unidade Saúde da Família Verdes Mares, neste primeiro semestre oportunizou conhecer o território e sua cartografia, identificando os Determinantes Sociais e correlacionando-os com o processo saúde-doença. Promoveu a integração com os profissionais da equipe, o conhecimento sobre o fluxograma descritor da Unidade e o processo de trabalho neste nível de Atenção. Além disso, planejaram ações, vivenciaram as consultas e as interconsultas, a visita domiciliar, a atuação do NASF em atividades coletivas, desenvolveram Educação em Saúde nos equipamentos sociais, com a construção de instrumentos educativos, vivenciaram o Grupo de Auriculoterapia e o Grupo de Idosos da Unidade, realizaram atividade de Educação Permanente na reunião de equipe, grupo de estudo e discussão de casos clínicos. O Grupo Tutorial - GT 4 desenvolveu a leitura e discussão de artigos científicos relacionados às temáticas e participaram da Mostra PET-Saúde Interprofissionalidade, socializando as experiências com os demais participantes do Projeto, na intencionalidade de aprender e compartilhar com os outros. 
Corroborando com os estudos apresentados por Forte et al (2016) estas atividades realizadas no PET-Saúde Interprofissionalidade também oportunizaram experiências interprofissionais, desenvolvendo habilidades para a prática colaborativa em equipe, para os estudantes dos cursos de graduação, para a preceptoria e para os profissionais do serviço, em busca da qualificação do cuidado em saúde.

\section{Alinhamento conceitual: correlação dos conceitos teóricos metodológicos da Educação Interprofissional e da Práticas Colaborativas com as atividades desenvolvidas no PET- Saúde Interprofissionalidade}

O Ministério da Saúde, incentivado pela agenda proposta pela Organização Pan-Americana da Saúde (OPAS), tem dado prioridade à inclusão da pauta da EIP em suas estratégias para o fortalecimento da política de formação profissional em saúde. Dentre as diversas parcerias intersetoriais, foi elaborado o Plano Nacional de Ação para Fortalecimento da Educação Interprofissional no Brasil (BRASIL, 2017b).

A estruturação deste Plano foi organizada em cinco linhas de ação aqui descritas: 1- Fortalecimento da EIP como dispositivo para a reorientação dos cursos de graduação em saúde, 2- Levantamento das iniciativas de EIP no Brasil, 3Desenvolvimento docente para a EIP, 4- Fortalecimento dos espaços de divulgação e produção do conhecimento em EIP e 5- EIP nos espaços de Educação Permanente em Saúde (FREIRE FILHO, 2019).

Sobre as ações a serem pensadas para a graduação em saúde nas três dimensões, o Plano Nacional de Ação para Fortalecimento da Educação Interprofissional no Brasil apresentou, dentre outras, o alinhamento conceitualmente às políticas de reorientação da formação e da atenção à saúde, na perspectiva de assegurar coerência teórico-conceitual e metodológica aos preceitos da interprofissionalidade, a compreensão do conceito de EIP e de trabalho interprofissional, suas bases teóricas e metodológicas, na dinâmica das graduações em saúde e a valorização da formação interprofissional em experiências de 
aprendizagem que fortaleçam o trabalho em equipe, tais como o PET-Saúde (BRASIL, 2017b).

De acordo com os pressupostos do PET-Saúde Interprofissionalidade, os grupos de aprendizagem tutorial do projeto devem proporcionar o desenvolvimento de competências colaborativa, tomando como referencial teórico-conceitual e metodológico a Educação Interprofissional, o Trabalho Colaborativo, o trabalho em equipe, o autocuidado e a autonomia das pessoas, famílias, grupos e comunidades (BRASIL, 2018b).

Buscando fortalecer o alinhamento conceitual sugerido no planejamento do Projeto e a necessidade de avaliação das atividades que estavam sendo realizadas, os participantes do PET-Saúde Interprofissionalidade, neste cenário de aprendizagem, identificaram a necessidade de organizar uma atividade que pudessem correlacionar os conceitos teóricos metodológicos da EIP e das PC com as atividades desenvolvidas no primeiro semestre de Projeto. Foi planejada então, a dinâmica da "colcha de retalhos", buscando, assim, um espaço de diálogo críticoreflexivo, com o compartilhar de saberes e uma aprendizagem significativa acerca da temática Educação Interprofissional.

Foi decidido, entre os estudantes e a preceptora, trazer para a USF as fotos das atividades vivenciadas neste território e em coletividade construírem a atividade de alinhamento conceitual.

No início da atividade na USF, a preceptora apresentou aos estudantes a história do livro A Colcha de Retalhos, de Conceil e Ribeiro (2010) que conta a narrativa dos personagens que, em meio à costura de uma colcha, feita com retalhos coloridos, vão resgatando memórias que fazem parte da identidade de cada um.

Uma vez imersos nesta narrativa, a preceptora apresentou também a sua colcha de retalhos, construída com diversos pedaços de tecido colorido, que traziam consigo lembranças de acontecimentos. Então, foi proposto aos participantes do PET-Saúde Interprofissionalidade a inserção dos registros fotográficos das atividades desenvolvidas, por ordem cronológica de sua realização neste território. À medida que a as fotos eram apresentadas e adicionadas à colcha de retalhos, os participantes traziam consigo as lembranças, as contribuições, o aprendizado de cada um nas vivências, bem como os desafios enfrentados. 
Em seguida, foram construídas targetas coloridas com os conceitos de Educação Interprofissional, práticas colaborativas, trabalho uniprofissional, multiprofissional, interprofissional, competências específicas, comuns e colaborativas, bem como conceitos acerca de comunicação interprofissional, cuidado centrado no paciente, clareza de papéis dos profissionais, funcionamento da equipe, resolução de conflitos e liderança colaborativa. Estas targetas foram colocadas sobre a colcha de retalhos, buscando correlacionar estes conceitos com as atividades realizadas, utilizando a metodologia ativa como estratégia pedagógica para a construção coletiva do conhecimento. Dessa forma, o alinhamento conceitual foi realizado de maneira dialógica, a partir do que foi vivenciado neste território, sendo esta, uma ação interprofissional fundamental para o desenvolvimento das atividades deste Projeto na Unidade Saúde da Família.

Como parte das atividades no GT, as Tutoras planejaram a socialização das vivências realizadas nas USF deste grupo de aprendizagem. A equipe do PETSaúde Interprofissionalidade da USF Integrada Verdes Mares desenvolveu esta mesma dinâmica da colcha de retalhos, ampliando, assim, os debates e as reflexões críticas através dos olhares e pensamentos dos demais participantes do Projeto, aqui representados pelas profissões de Educação Física, Nutrição, Fonoaudiologia, Medicina, Terapia Ocupacional e Odontologia.

Dessa experiência, veio o compartilhar de saberes e, assim como a beleza visual da colcha de retalhos, tão colorida em sua essência, estaria a beleza e a diversidade de pensamentos, na ação de aprender com os outros, sobre os outros e entre si, contextualizados com o território e com a comunidade adstrita. Reeves (2016b) destaca em seu texto, que métodos de aprendizado que permitem interatividade são, portanto, uma característica chave para EIP.

As metodologias ativas utilizam a problematização como principal estratégia de ensino- aprendizagem, buscando motivar os alunos, uma vez que, diante do problema, estes se debruçam, examinam, fazem reflexões, relacionam a sua história e passam a ressignificar suas descobertas (MITRE et al 2008). Outrossim, estas metodologias trazem oportunidades de ensino em que os alunos passam a ter um comportamento mais ativo, envolvendo-os de modo que sejam mais engajados, desenvolvendo atividades que possam auxiliar o estabelecimento de relações com o 
contexto, o desenvolvimento de estratégias cognitivas e o processo de construção de conhecimento (VALENTE; ALMEIDA; GERALDINI, 2017).

Independentemente do tempo de duração, a EIP sempre deverá ser incluída na formação em saúde, dada a sua importância para o desenvolvimento de competências para a educação e 0 trabalho interprofissional: competências específicas de cada profissão, competências comuns a todas as profissões e competências colaborativas, necessárias para a reconfiguração das relações profissionais em benefício da qualidade da atenção à saúde (BARR, 1998).

Forte et al (2016) descreve que o estímulo a práticas interprofissionais, é uma proposta de aprendizado para o enfrentamento de um sistema de saúde fragmentado, pois, estando coeso e em diálogo, em um movimento de construção coletiva, o grupo tutorial pode compreender sobre a potencialidade do trabalho em equipe e favorece ao aprendizado colaborativo.

Portanto, como destaca Costa (2016) é necessário, reforçar os desafios impostos, os quais apontam para a busca de apoios institucionais, respaldados nas políticas atuais e futuras, da qualificação do corpo docente para a EIP, no fortalecimento das relações entre universidade, serviços e comunidades, da necessidade de investimento na mudança das relações interprofissionais e interpessoais dos muitos atores envolvidos na formação e na produção dos serviços de saúde, entre outros.

\section{CONCLUSÃO}

O surgimento de espaços de diálogo nos cenários de aprendizagem nas Unidades Saúde da Família proporcionados por programas como o PET-Saúde Interprofissionalidade permitiu aos tutores, preceptores e estudantes dialogarem sobre casos clínicos, nos grupos de estudos, nos planejamentos, na execução das ações e nos processos avaliativos, contribuindo para a formação em saúde. A inserção de maneira programada, proposital e sistematizada destes participantes desenvolvendo ações com as diversas profissões e a realização da dinâmica da 
"colcha de retalhos" aprimorou o conhecimento, através de uma aprendizagem significativa em suas construções, permitindo uma análise da realidade vivenciada, buscando superar as fragilidades impostas nos serviços.

Vivenciar a experiência em grupos tutoriais de aprendizagem, ressignifica a prática da preceptoria, através de novas estratégias com metodologias ativas de ensino aprendizagem, construindo assim, iniciativas exitosas em Educação Interprofissional e Práticas Colaborativas.

Este trabalho também traz a reflexão da necessidade de fortalecer a inserção da comunidade nas atividades dos serviços de saúde, o que se apresenta como um desafio a ser superado, dada a importância desta articulação para dar resolutividade às necessidades e aos problemas de saúde cada vez mais complexos no cuidado em saúde.

Percebe-se, então, que experiências desta natureza, devem ser estimuladas, como frente a necessidade de aproximar, alinhar e implementar os conceitos teóricos metodológicos da EIP e PC na formação e no trabalho em saúde, qualificando desta forma, a atenção em saúde da comunidade e fortalecendo a integração ensino-serviço.

\section{REFERÊNCIAS BIBLIOGRÁFICAS}

BARR, H. Competent to collaborate: Towards a competency-based model for interprofessional education. Journal of Interprofessional Care, v. 12, n. 2, p. 181187, 1998.

BARR, H.; LOW, H. Introdução à Educação Interprofissional. Fareham: CAIPE, 2013.

BATISTA, N. A. Educação Interprofissional em Saúde: Concepções e Práticas. Caderno FNEPAS, olume 2 - Janeiro 2012.

BRASIL. Constituição da República Federativa do Brasil: texto constitucional promulgado em 5 de outubro de 1988, com alterações determinadas pelas Emendas Constitucionais $n^{\circ} 1 / 92$ a 91/2016 e pelo Decreto Lesgilativo $n^{\circ}$ 186/2008. - Brasília: Senado Federal, Coordenações de Edições Técnicas, 2016.

BRASIL. Construindo caminhos possíveis para a Educação Interprofissional em Saúde nas Instituições de Ensino Superior do Brasil / Ministério da Saúde, Secretaria de Gestão do Trabalho e da Educação na Saúde, Departamento de Gestão da Educação na Saúde - 1. ed. rev. - Brasília: Ministério da Saúde, 2018a.

BRASIL. Edital $n^{\circ}$ 10, 23 de julho 2018. Seleção para o programa de educação pelo trabalho para a saúde pet-saúde/interprofissionalidade - 2018/2019. Brasília (DF): Ministério da Saúde; 
2018b.

BRASIL. Ministério da Saúde e Ministério da Educação. Portaria Interministerial de $n^{\circ} 422$, de 3 de março de 2010. Institui o Programa de Educação pelo Trabalho para a Saúde (PETSaúde) e dá outras providências, 2010.

BRASIL. Ministério da Saúde. Portaria ${ }^{\circ}$ 2.436, de 21 de setembro de 2017. Aprova a Política Nacional de Atenção Básica, estabelecendo a revisão de diretrizes para a organização da Atenção Básica, no âmbito do Sistema Único de Saúde (SUS), 2017a.

BRASIL. Ministério da Saúde. Portaria $n^{\circ} 306$, de 22 de outubro de 2018. Dispõe sobre o resultado preliminar da seleção de projetos para o PET-Saúde interprofissionalidade 20182019 nos termos do edital $n^{\circ}$ 10, 23 de julho 2018. Brasília (df): ministério da saúde; 2018c.

BRASIL. Ministério da Saúde. Relatório Final da Oficina de Alinhamento Conceitual sobre Educação e Trabalho Interprofissional em Saúde. Brasília, DF: Ministério da Saúde; 2017b.

CAIPE. Centre for the Advancement of Interprofessional Education. United Kingdom, 2002.

CANADIAN INTERPROFESSIONAL HEALTH COLLABORATIVE (CIHC). A national interprofessional competency framework. Vancouver, BC: Canadian Interprofessional Health Collaborative, 2010.

CONCEIL, C. da S.; NYE, R. A. A Colcha de Retalhos. Ilustrações Ellen Pestili, Editora do Brasil, 2010.

COSTA, M. V. da. A educação interprofissional no contexto brasileiro: algumas reflexões. Interface Comunicação Saúde Educação, 2016; 20(56):197-8.

COSTA, M. V. da; Peduzzi, M.; Freire Filho, j.r.; Silva, C. B. G. Educação Interprofissional em Saúde. - Natal: SEDIS-URFN, 2018.

FORTE, F. D. S.; Morais, H. G. F.; Rodrigues, S. A. G.; Santos, J. S.; Oliveira, P. F.; Morais, M. S. T.; et al. Educação interprofissional e o Programa de Educação pelo Trabalho para a Saúde/Rede Cegonha: potencializando mudanças na formação acadêmica. Interface. Comunicação Saúde Educação, 2016; 20(58):787-96.

FREIRE, FILHO JR.; SILVA, C. B. G.; COSTA, M. V.; FORSTER, A. C. Educação Interprofissional nas políticas de reorientação da formação profissional em saúde no Brasil. Saúde Debate/ Rio de Janeiro, V. 43, N. Especial 1, P. 86-96, AGO 2019.

MADRUGA, L. M. S.; RIBEIRO, K. S. Q. S.; FREITAS, C. H. M.; PÉREZ, I. A. B.; PESSOA, T. R. R. F.; BRITO, G. E. G. O PET-Saúde da Família e a Formação de Profissionais da Saúde: percepção dos estudantes. Interface. Comunicação Saúde Educação, .2015; 19 Supl 1:805-16.

MENDES, E. V. Desafios do SUS. Brasília, DF: CONASS, 2019.

MITRE, S. M.; SIQUEIRA-BATISTA, R.; GIRARDIDE MENDONÇA, J. M.; MORAIS- PINTO, N. M.; MEIRELLES, C. A. B.; PINTO-PORTO, C.; MOREIRA, T.; HOFFMANN, L. M. A. Metodologias ativas de ensino-aprendizagem na formação profissional em saúde: debates atuais. Ciência e Saúde Coletiva, Rio de Janeiro, v. 13, 2008.

ORGANIZAÇÃO MUNDIAL DE SAÚDE. Marco para Ação em Educação Interprofissional e Prática Colaborativa. Genebra: OMS; Editora Freelance, 2010.

PEDUZZI, M. O SUS é Interprofissional. Interface. Comunicação Saúde Educação. 2016; 20(56):199-201. 
REEVES, S. et al. A BEME systematic review of the effects of interprofessional education: BEME Guide No. 39. Med Teach, v. 38, n. 7, p. 656-68, 2016a.

REEVES, S. Porque precisamos da educação interprofissional para um cuidado efetivo e seguro. Interface. Comunicação, Saúde, Educação, v. 20, n. 56, p. 185-197, 2016b.

VALENTE, V. A.; ALMEIDA, M. E. B.; GERALDINI, A. F. S. Metodologias ativas: das concepções às práticas em distintos níveis de ensino. Rev. Diálogo Educ., Curitiba, v. 17, n. 52, p. 455-478, abr./jun. 2017. 